\title{
THE EFFECT OF PREGNANCY ON RENAL FUNCTION IN WOMEN WITH PRE-EXISTING ESSENTIAL HYPERTENSION AND WITH CHRONIC DIFFUSE GLOMERULONEPHRITIS ${ }^{1}$
}

\author{
BY IRWIN WELLEN, CATHERINE A. WELSH, AND HOWARD C. TAYLOR, JR., \\ WITH THE TECHNICAL ASSISTANCE OF ANNA ROSENTHAL \\ (From the Department of Obstetrics and Gynecology, New York University College of Medicine, and \\ the Obstetrical and Gynecological Service of the Third (New York University) Surgical \\ Division of Bellevue Hospital, New York City)
}

(Received for publication February 24, 1944)

The effect of pregnancy on the renal function of patients with essential hypertension or chronic glomerulonephritis has been the object of the investigation to be reported here. The study has involved the application of renal clearance methods to a group of patients, during their pregnancies and repeatedly during the 1 to 4 years after their delivery. In 3 patients, the series of records includes also observations made before conception. Such repeated studies were designed firstly to demonstrate the immediate changes in renal function developing during the period of gestation; and secondly, to determine whether, after pregnancy is over, there has occurred any significant acceleration in the course of the primary disease.

These patients with pre-existing renal or vascular disease are to be contrasted with the cases of specific "toxemia" or eclampsia, upon which we have previously published observations. In our first report on this subject, studies based upon inulin and diodrast clearances showed that the glomerular filtration rate, effective renal blood flow, and tubular excretory mass were unaffected in normal pregnancy (1). In a second paper (2), it was shown that in patients with specific toxemia, the glomerular filtration rate was slightly diminished and the effective renal blood flow increased during the latter part of pregnancy. Following delivery, the blood flow became normal in about half of these patients, while in the others it fell to levels indicative of slight renal ischemia. The latter trend occurred in those women in whom hypertension became permanently established after their attack of toxemia of pregnancy. These observations are in general similar to the

\footnotetext{
1 This study was made with the aid of a grant from the Commonwealth Fund.
}

reported work of other investigators using these methods in cases of toxemia of pregnancy.

Renal function studies of this type have also been made previously in pregnancy complicated by essential hypertension $(3,4)$. There are, however, no published observations of effective renal blood flow, diodrast $\mathrm{Tm}$, and glomerular filtration rate made before, during, and after pregnancy in the same patient. The studies to be reported are also apparently the first of their kind concerning the effects of pregnancy on chronic diffuse glomerulonephritis.

\section{METHOD}

Several aspects of renal function were studied by the clearance principle used in our previous studies. The rate of glomerular filtration was measured at first by the inulin clearance. Later mannitol, whose clearance is identical with that of inulin, was substituted (5). The effective renal blood flow was determined by the diodrast clearance except in the first 2 pregnancies observed in the essential hypertension group. In these, changes in renal blood flow were followed by the phenol red clearance. The latter gives figures which average about two-thirds of the diodrast clearance, but this ratio is not constant and we have not attempted to present actual figures for blood flow computed from the phenol red clearance. Nevertheless, comparison of figures for phenol red clearance in successive observations on the same individual gives a basis for detecting changes in the renal blood flow in that patient. The maximum capacity of the tubules to excrete diodrast was measured by the clearance of diodrast at high levels of plasma concentration. This function, referred to here as diodrast $\mathrm{Tm}$, is regarded as a measure of the mass of functioning tubular tissue (6).

The preparation of the patients for the tests, the collection of the specimens, and the analytical procedures used were those previously described $(1,2)$. In the cases of this series, as in those already reported, no observation in which the urine flow was below $2 \mathrm{cc}$. per minute was considered acceptable. Our experience has convinced us that observations made where the urine flow is below this level are of no value in pregnant women, in whom complete emptying of the bladder is especially difficult. 


\section{MATERIAL}

Essential hypertension. Observations were made during and after the 7 pregnancies of 6 women suffering from preexisting hypertension. In 3 of these, who had been patients at the New York University Hypertension and Nephritis Clinic, renal function studies had been made before the advent of pregnancy. After delivery, the patients were followed by further tests giving periods of post-partum observation which varied in duration from 1 to 4 years.

That these were actually cases of uncomplicated essential hypertension and not of primary renal disease is shown by the history of the patients. In none had there been edema, hematuria, or history of acute diffuse glomerulonephritis. In 1 patient whom we have classed in this group, there had been slight proteinuria in addition to the hypertension for 8 years following a pregnancy complicated by eclampsia in 1933. The possibility exists then that this case only should have been classified in the other group. The duration of observed hypertension in these patients before conception is shown in Table $I$.

Glomerulonephritis. Two women with diffuse glomerulonephritis became pregnant while under observation in the New York University Hypertension and Nephritis Clinic. These patients were known to have exhibited edema, hypertension, proteinuria, and hematuria for several months before their pregnancies began. Urea clearances determined before conception indicated a reduction to about one-half the normal value In one (J. G.) and to two-thirds the normal in the other (P. R.).

Controls. The figures taken as normal for the respective functions studied are made up from the averages obtained from 14 non-pregnant women observed by others $(7,8)$ and 8 pregnant women observed by us (1). The combination of these groups was considered justifiable since the average figures for the two, the pregnant and non-pregnant women, were practically identical. The following figures, corrected to a surface area of 1.73 square meters, are the averages for the 22 normal women: inulin clearance, 122 ml. per minute; diodrast clearance, $613 \mathrm{ml}$. per minute; diodrast $\mathrm{Tm}, 43.7 \mathrm{mgm}$. iodine per minute; filtration fraction, 19.9 per cent; $C_{D} / T_{m D}$ ratio, 14.0 and $C_{I N} / T_{m D}$, ratio 2.79 .

\section{RESULTS}

Results are to be considered from two points of view. First, there is the immediate effect of pregnancy on the kidney function of the woman already suffering from chronic renal or vascular disease. Secondly, there is the debated question of whether pregnancy is permanently detrimental to the kidney function of women with one of these diseases. These two aspects must be considered separately in relation to essential hypertension and to chronic glomerulonephritis since current opinion makes a sharp distinction between these two diseases.

The figures for the 48 observations are given in detail in Tables II and III. In these tables, each plasma clearance figure represents the average of 3 urine collection periods; each diodrast Tm figure, the average of 5 urine collection periods.

TABLE I

Clinical data

\begin{tabular}{|c|c|c|c|c|c|c|c|c|c|c|c|c|c|c|c|c|}
\hline & \multirow[b]{2}{*}{ Subject } & \multirow[b]{2}{*}{ Age } & \multirow[b]{2}{*}{$\begin{array}{c}\text { Par- } \\
\text { ity }\end{array}$} & \multicolumn{3}{|c|}{ Before conception } & \multicolumn{4}{|c|}{ Pregnancy } & \multicolumn{3}{|c|}{ Delivery } & \multicolumn{3}{|c|}{$\begin{array}{l}\text { Postpartum } \\
\text { blood pressure range }\end{array}$} \\
\hline & & & & $\begin{array}{c}\text { Dura- } \\
\text { tion of } \\
\text { observed } \\
\text { hyper- } \\
\text { tension }\end{array}$ & $\begin{array}{r}\text { Blood p } \\
\text { ran }\end{array}$ & $\begin{array}{l}\text { ressure } \\
\text { ige }\end{array}$ & Blood r & $\begin{array}{l}\text { ressure } \\
\text { ige }\end{array}$ & $\begin{array}{l}\text { Protei- } \\
\text { nuria }\end{array}$ & Edema & Date & $\begin{array}{c}\text { Week } \\
\text { of } \\
\text { ges- } \\
\text { tation }\end{array}$ & Baby & $\begin{array}{c}\text { Dura- } \\
\text { tion of } \\
\text { follow- } \\
\text { up }\end{array}$ & $\begin{array}{r}\text { Blood p } \\
\text { ran }\end{array}$ & $\begin{array}{l}\text { ressure } \\
\text { ge }\end{array}$ \\
\hline $\begin{array}{l}1 \\
2 \\
3 \\
4 \\
4 \\
5 \\
6 \\
7 \\
8\end{array}$ & $\begin{array}{l}\left.\begin{array}{l}\text { L. L. } \\
\text { J. G. } \\
\text { M. J. } \\
\text { M. S. }\end{array}\right\} \\
\text { H. F. } \\
\text { H. M. } \\
\text { P. R. } \\
\text { J. Go. }\end{array}$ & $\begin{array}{l}42 \\
31 \\
37 \\
32 \\
34 \\
28 \\
39 \\
34 \\
23\end{array}$ & $\begin{array}{l}4 \\
1 \\
1 \\
1 \\
1 \\
2 \\
1 \\
4 \\
2 \\
1\end{array}$ & $\begin{array}{c}0 \\
0 \\
3 \text { years } \\
0 \\
2 \text { years } \\
2 \text { years } \\
8 \text { years } \\
8 \text { mos. } \\
2 \text { years }\end{array}$ & $\begin{array}{l}138 / 110 \\
144 / 106 \\
134 / 100 \\
180 / 94 \\
124 / 92 \\
150 / 110\end{array}$ & $\begin{array}{l}170 / 105 \\
204 / 118 \\
192 / 130 \\
220 / 130 \\
150 / 102 \\
226 / 150\end{array}$ & $\begin{array}{l}150 / 94 \\
130 / 90 \\
136 / 98 \\
132 / 96 \\
136 / 100 \\
158 / 98 \\
120 / 80 \\
160 / 110\end{array}$ & $\begin{array}{l}210 / 130 \\
178 / 110 \\
160 / 100 \\
164 / 110 \\
160 / 120 \\
220 / 120 \\
166 / 114 \\
220 / 150\end{array}$ & $\begin{array}{r}0 \\
0 \\
++ \\
+++\end{array}$ & $\begin{array}{l}\mathbf{0} \\
\mathbf{0} \\
\mathbf{0} \\
\mathbf{0} \\
\mathbf{0} \\
+ \\
\mathbf{0} \\
\mathbf{0}\end{array}$ & $\begin{array}{r}7-24-38 \\
3-11-39 \\
5-19-40 \\
11-9-38 \\
6-7-41 \\
5-12-42 \\
6-9-41 \\
9-13-41 \\
7-29-42\end{array}$ & $\begin{array}{l}36 \\
40 \\
22 \\
40 \\
40 \\
40 \\
40 \\
40 \\
36\end{array}$ & $\begin{array}{l}\text { Dead } \\
\text { Live } \\
\text { Dead } \\
\text { Live } \\
\text { Live } \\
\text { Live } \\
\text { Live } \\
\text { Live } \\
\text { Live }\end{array}$ & $\begin{array}{l}1 \text { mo. } \\
1 \text { mo. } \\
3 \text { years } \\
41 \text { yrs. } \\
2 \text { years } \\
1 \text { year } \\
2 \text { years } \\
2 \text { years } \\
1 \text { year }\end{array}$ & $\begin{array}{l}166 / 105 \\
148 / 94 \\
160 / 110 \\
152 / 102 \\
130 / 100 \\
132 / 100 \\
160 / 108 \\
130 / 94 \\
160 / 120\end{array}$ & $\begin{array}{l}180 / 114 \\
164 / 90 \\
240 / 140 \\
170 / 110 \\
200 / 120 \\
160 / 114 \\
214 / 130 \\
160 / 110 \\
210 / 150\end{array}$ \\
\hline
\end{tabular}

L. L. Hypertension noted when the patient was first seen in the second month of her pregnancy.

J.G. Hypertension present when the patient was first seen in the sixth week of her pregnancy.

M. J. Hypertension has persisted since toxemia of pregnancy in 1937 .

M.S. Hypertension reported to have been present since 1937 .

H. F. Hypertension has persisted since toxemia of pregnancy in 1937.

H. M. Hypertension and proteinuria first noted in 1933, and found at all observations since that time.

P. R. In April 1940, this patient had acute diffuse glomerulonephritis, either an initial attack or an acute exacerbation. Since that time she has had hypertension, proteinuria, and hematuria, and on occasions edema.

J. Go. This patient was first seen in January 1940, in the fourth month of pregnancy, with hypertension, proteinuria, and edema. She was delivered at the seventh month of a premature baby who died. Since that time, she has had hypertension, proteinuria, and hematuria. 
Clinical effects of pregnancy on essential hypertension

There are several clinical reports which show that women with essential hypertension are especially liable to develop signs of "superimposed" toxemia if they become pregnant (9 to 11). It was found (12) in 63 per cent of one series of 122 cases that the hypertension was exaggerated at least temporarily during the latter months of pregnancy and in 70 per cent, proteinuria appeared. A report (13) from the New
York University Obstetrical Service at Bellevue states that in 59 of 69 pregnancies in women with pre-existing essential hypertension, there developed signs of superimposed toxemia. At the same time, there are certainly cases in which no exaggeration of the hypertension occurs, one report (9) placing the figure for this favorable course at 50 per cent. Indeed, there may actually be a striking temporary decrease in blood pressure during the middle trimester $(12,14)$. The significance of this is not clear, although it may be ascribed simply to an exaggeration of

TABLE II

Essential hypertension

\begin{tabular}{|c|c|c|c|c|c|c|c|c|c|c|c|c|c|c|c|c|c|c|}
\hline \multirow{2}{*}{ ject } & \multirow{2}{*}{$\begin{array}{l}\text { Anto- } \\
\text { par- } \\
\text { tum } \\
\text { or } \\
\text { post- } \\
\text { par- } \\
\text { tum }\end{array}$} & \multirow{2}{*}{$\begin{array}{l}\text { Sur- } \\
\text { face } \\
\text { area }\end{array}$} & \multirow{2}{*}{ Date } & \multirow{2}{*}{$\begin{array}{l}\text { Dura- } \\
\text { tion } \\
\text { of } \\
\text { preg- } \\
\text { nanoy } \\
\text { by } \\
\text { his } \\
\text { tory }\end{array}$} & \multirow{2}{*}{$\begin{array}{l}\text { Thme } \\
\text { before } \\
\text { or } \\
\text { after } \\
\text { do- } \\
\text { livery }\end{array}$} & \multirow{2}{*}{$\begin{array}{c}\text { Blood } \\
\text { pres } \\
\text { sure } \\
\text { during } \\
\text { teat }\end{array}$} & \multirow{2}{*}{ Protein- } & \multicolumn{3}{|c|}{ Plasma olearances } & \multirow{2}{*}{\begin{tabular}{|c|} 
Effoc \\
tive \\
renal \\
blood \\
flow
\end{tabular}} & \multirow{2}{*}{$\mid \begin{array}{c}\text { Innoling } \\
\text { Phenol } \\
\text { red }\end{array}$} & \multirow{2}{*}{$\begin{array}{c}\text { Filtra- } \\
\text { tion } \\
\text { fraction }\end{array}$} & \multirow{2}{*}{$\begin{array}{l}\text { Phenol } \\
\text { red/ } \\
\text { Diodrast }\end{array}$} & \multirow{2}{*}{$\begin{array}{c}\text { Diodraat } \\
\text { Tm }\end{array}$} & \multirow{2}{*}{$\mid \begin{array}{c}\text { Effoo- } \\
\text { tive } \\
\text { renal } \\
\text { blood } \\
\text { flow! } \\
\text { Tmp } \\
\text { A }\end{array}$} & \multirow{2}{*}{$\begin{array}{c}C_{D} / \\
\operatorname{Tm}_{D} \\
A\end{array}$} & \multirow{2}{*}{$\begin{array}{c}\text { CIN/ } \\
\text { TmD } \\
\\
\text { A }\end{array}$} \\
\hline & & & & & & & & $\begin{array}{l}\text { Inv- } \\
\text { lin }\end{array}$ & $\begin{array}{l}\text { Pho- } \\
\text { nol } \\
\text { red }\end{array}$ & $\begin{array}{l}\text { Dio- } \\
\text { drast }\end{array}$ & & & & & & & & \\
\hline L. L. & $\begin{array}{l}\text { A-P } \\
\mathbf{P}-\mathbf{P}\end{array}$ & m. & $\begin{array}{l}\text { June 2, } 1938 \\
\text { June 23, } 1938 \\
\text { July 14, } 1938 \\
\text { Auguat 5, 1938 }\end{array}$ & $\begin{array}{l}28 \\
31 \\
34\end{array}$ & $\begin{array}{l}8 \\
5 \\
2 \\
2\end{array}$ & $\begin{array}{l}152 / 118 \\
170 / 130 \\
100 / 114 \\
170 / 100\end{array}$ & $\begin{array}{l}0 \\
0 \\
0 \\
0\end{array}$ & $\begin{array}{l}197 \\
198 \\
181 \\
178\end{array}$ & $\begin{array}{l}399 \\
626 \\
661 \\
461\end{array}$ & $q . m$. & $\left|\begin{array}{c}m l . p e r \\
1.75 \\
o q . m . \\
\text { per } \\
\text { minute }\end{array}\right|$ & \begin{tabular}{|c|} 
per \\
cent \\
\\
49.4 \\
37.7 \\
27.4 \\
38.7 \\
\end{tabular} & $\begin{array}{c}\text { per } \\
\text { cent }\end{array}$ & $\begin{array}{c}\text { por } \\
\text { cont }\end{array}$ & $\begin{array}{l}\text { mom. } \\
\text { iodins } \\
\text { per } \\
\text { minute }\end{array}$ & & & \\
\hline J.G. & $\begin{array}{l}\text { A-P } \\
\mathbf{P}-\mathbf{P}\end{array}$ & 1.73 & $\begin{array}{l}\text { Fobruary 24, } 1939 \\
\text { March 10, } 1939 \\
\text { March 21, } 1939\end{array}$ & $\begin{array}{l}88 \\
40\end{array}$ & $\begin{array}{l}2 \\
0 \\
2\end{array}$ & $\begin{array}{l}170 / 110 \\
158 / 110 \\
160 / 98\end{array}$ & $\begin{array}{l}0 \\
0 \\
0\end{array}$ & $\begin{array}{r}98 \\
103 \\
100\end{array}$ & $\begin{array}{l}298 \\
310\end{array}$ & $\begin{array}{l}460 \\
524 \\
378\end{array}$ & $\begin{array}{l}748 \\
874 \\
500\end{array}$ & $\begin{array}{l}32.9 \\
32.3\end{array}$ & $\begin{array}{l}21.3 \\
19.7 \\
26.5\end{array}$ & $\begin{array}{l}68.4 \\
82.0\end{array}$ & $\begin{array}{l}40.1 \\
44.9 \\
46.6\end{array}$ & $\begin{array}{l}17.0 \\
19.9 \\
11.4\end{array}$ & $\begin{array}{r}10.5 \\
11.9 \\
8.6\end{array}$ & $\begin{array}{l}2.23 \\
2.34 \\
2.28\end{array}$ \\
\hline M.J. & $\mathbf{P}-\mathbf{P}$ & 1.58 & $\begin{array}{l}\text { Fobruary 21, 1938* } \\
\text { March 21, } 1838^{*} \\
\text { March 29, } 1940 \\
\text { May 15, } 1940 \\
\text { June 13, 1940 } \\
\text { April } 4,1941^{*} \\
\text { May 7, } 1941^{*} \\
\text { May 24, } 1943\end{array}$ & $\begin{array}{l}15 \\
22\end{array}$ & $\begin{array}{l}7 \\
0 \\
4\end{array}$ & $\begin{array}{l}162 / 110 \\
176 / 114 \\
180 / 116 \\
194120 \\
152 / 98 \\
208 / 120\end{array}$ & $\begin{array}{l}0 \\
0 \\
0 \\
0 \\
0 \\
0\end{array}$ & \begin{tabular}{|c|}
118 \\
132 \\
133 \\
121 \\
92 \\
105 \\
84 \\
$89 \mathrm{~m}$
\end{tabular} & $\begin{array}{l}291 \\
402 \\
384 \\
339 \\
257 \\
\\
312\end{array}$ & $\begin{array}{l}485 \\
531 \\
610 \\
465 \\
349 \\
461 \\
570 \\
541\end{array}$ & $\begin{array}{r}824 \\
848 \\
1046 \\
740 \\
498 \\
752 \\
877 \\
718\end{array}$ & $\begin{array}{l}40.2 \\
32.9 \\
34.7 \\
35.7 \\
35.8 \\
\\
\\
28.6\end{array}$ & $\begin{array}{l}24.3 \\
24.9 \\
21.8 \\
26.0 \\
26.4 \\
22.8 \\
14.7 \\
19.8\end{array}$ & $\begin{array}{l}60.6 \\
75.7 \\
63.0 \\
72.9 \\
73.7\end{array}$ & $\begin{array}{l}41.1 \\
41.3 \\
44.2 \\
38.9 \\
35.9 \\
45.1 \\
47.5\end{array}$ & $\begin{array}{l}19.6 \\
20.4 \\
24.9 \\
17.6 \\
11.9 \\
17.9 \\
20.9 \\
17.1 \\
\end{array}$ & \begin{tabular}{|r|}
11.6 \\
12.7 \\
14.5 \\
11.1 \\
8.3 \\
11.0 \\
13.6 \\
12.9
\end{tabular} & $\begin{array}{l}2.81 \\
3.14 \\
3.17 \\
2.88 \\
2.19 \\
2.50 \\
2.00 \\
2.12\end{array}$ \\
\hline M. 8 . & $\begin{array}{l}\text { A-P } \\
\text { P-P }\end{array}$ & 1.63 & $\begin{array}{l}\text { Ootober } 18,1938 \\
\text { November 16, } 1938 \\
\text { November 21, } \\
\text { Docembermber 28, } 1938 \\
\text { May 2, 1939, } \\
\text { Pobruary 6, } 1941 \\
\text { April 10, 1941 } \\
\text { June 20,1941 } \\
\text { January 14, 1942 } \\
\text { May 7, } 1943\end{array}$ & $\begin{array}{l}37 \\
40\end{array}$ & $\begin{array}{r}3 \\
0 \\
1 \\
6 \\
6 \\
19 \\
10 \\
2\end{array}$ & $\begin{array}{l}120 / 80 \\
200 / 108 \\
200 / 118 \\
200 / 128 \\
198 / 118 \\
130 / 86 \\
142 / 96 \\
126 / 92 \\
150 / 100 \\
178 / 108\end{array}$ & $\begin{array}{l}0 \\
0 \\
0 \\
0 \\
0 \\
0 \\
0 \\
0 \\
0 \\
0\end{array}$ & $\begin{array}{l}190 \\
138 \\
122 \\
122 \\
103 \\
117 \\
111 \\
104 \mathrm{~m} \\
115 \mathrm{~m} \\
104 \mathrm{~m}\end{array}$ & \begin{tabular}{|l}
535 \\
375 \\
321 \\
295 \\
257 \\
319 \\
420 \\
363 \\
322 \\
318
\end{tabular} & $\begin{array}{l}387 \\
624 \\
706 \\
590 \\
652 \\
448\end{array}$ & $\begin{array}{r}661 \\
934 \\
1025 \\
1030 \\
1034 \\
720\end{array}$ & $\begin{array}{l}35.5 \\
36.8 \\
38.0 \\
41.4 \\
40.1 \\
36.7 \\
33.6 \\
28.7 \\
35.7 \\
32.7\end{array}$ & $\begin{array}{l}26.6 \\
18.8 \\
19.9 \\
17.6 \\
17.7 \\
23.5\end{array}$ & $\begin{array}{l}66.4 \\
51.2 \\
59.5 \\
61.5 \\
49.4 \\
71.6\end{array}$ & $\begin{array}{l}51.8 \\
58.4 \\
54.5 \\
46.0\end{array}$ & $\begin{array}{l}12.6 \\
17.7 \\
19.5 \\
19.6 \\
19.6 \\
13.7\end{array}$ & $\begin{array}{r}7.3 \\
11.8 \\
13.4 \\
11.2 \\
12.4 \\
8.5\end{array}$ & $\begin{array}{l}1.95 \\
2.22 \\
2.67 \\
1.97 \\
2.18 \\
1.97\end{array}$ \\
\hline H. F. & $\begin{array}{l}\text { A-P } \\
\text { P-P }\end{array}$ & 1.44 & $\begin{array}{l}\text { Fobruary 27, } 1939 \\
\text { November } 29,1939 \\
\text { January 28, } 1942 \\
\text { March 26, } 1942 \\
\text { May 8, } 1942 \\
\text { May 28, } 1942 \\
\text { June 14, } 1943 \\
\end{array}$ & $\begin{array}{l}25 \\
33 \\
40\end{array}$ & $\begin{array}{r}15 \\
7 \\
0 \\
2\end{array}$ & $\begin{array}{l}192 / 134 \\
150 / 106 \\
130 / 94 \\
140 / 100 \\
190 / 130 \\
15 / 100 \\
140 / 100\end{array}$ & $\begin{array}{l}\mathbf{0} \\
\mathbf{0} \\
\mathbf{0} \\
\mathbf{0} \\
\mathbf{0} \\
\mathbf{0}\end{array}$ & $\begin{array}{l}121 \\
125 \\
131 \mathrm{~m} \\
148 \mathrm{~m} \\
103 \mathrm{~m} \\
11 \mathrm{~m} \\
93 \mathrm{~m}\end{array}$ & $\begin{array}{l}319 \\
329 \\
420 \\
380 \\
304 \\
337 \\
839 \\
\end{array}$ & $\begin{array}{r}494 \\
596 \\
1006 \\
672 \\
505 \\
508 \\
478\end{array}$ & $\begin{array}{r}856 \\
983 \\
1444 \\
975 \\
817 \\
864 \\
787\end{array}$ & $\begin{array}{l}37.9 \\
33.0 \\
31.2 \\
39.0 \\
33.9 \\
33.0 \\
27.4\end{array}$ & $\begin{array}{l}24.5 \\
21.0 \\
112.0 \\
22.0 \\
18.2 \\
18.6 \\
19.4\end{array}$ & $\begin{array}{l}64.6 \\
55.2 \\
41.7 \\
56.5 \\
55.8 \\
56.4 \\
71.0\end{array}$ & $\begin{array}{l}42.6 \\
36.3 \\
45.3 \\
49.2 \\
4.8 \\
40.1 \\
40.5\end{array}$ & $\begin{array}{l}20.1 \\
23.0 \\
33.8 \\
22.8 \\
19.1 \\
20.2 \\
18.4\end{array}$ & $\begin{array}{l}11.6 \\
14.0 \\
22.5 \\
15.7 \\
11.8 \\
11.0 \\
11.2\end{array}$ & $\begin{array}{l}2.83 \\
2.93 \\
3.07 \\
8.47 \\
2.41 \\
2.60 \\
2.18\end{array}$ \\
\hline H. M.† & $\mathbf{A}-\mathbf{P}$ & 1.82 & $\begin{array}{l}\text { March 8, } 1941 \\
\text { March 12, } 1941 \\
\text { March 19, } 1941 \\
\text { April 2, 1941 } \\
\text { May 12, 1941 } \\
\text { June 16, 1941 } \\
\text { June 28, } 1943\end{array}$ & $\begin{array}{l}27 \\
28 \\
29 \\
32 \\
37\end{array}$ & $\begin{array}{c}13 \\
12 \\
11 \\
8 \\
3 \\
1 \\
1\end{array}$ & $\begin{array}{l}210 / 110 \\
180 / 106 \\
100 / 116 \\
160 / 98 \\
200 / 120 \\
198 / 148 \\
190 / 110\end{array}$ & $\begin{array}{l} \pm \\
\pm \\
+ \\
+ \\
+\end{array}$ & \begin{tabular}{|l|}
131 \\
136 \\
122 \\
113 \\
102 \\
82 \\
$101 \mathrm{~m}$
\end{tabular} & & $\begin{array}{l}810 \\
680 \\
640 \\
656 \\
684 \\
381 \\
441\end{array}$ & $\begin{array}{r}1222 \\
892 \\
950 \\
965 \\
870 \\
584 \\
642\end{array}$ & & $\begin{array}{l}16.1 \\
20.1 \\
19.2 \\
17.4 \\
17.4 \\
20.5 \\
22.9\end{array}$ & & $\begin{array}{l}52.1 \\
51.2\end{array}$ & $\begin{array}{l}22.6 \\
19.2 \\
18.4 \\
18.7 \\
16.8 \\
11.3 \\
12.4\end{array}$ & \begin{tabular}{r|}
15.7 \\
13.1 \\
12.4 \\
12.7 \\
11.3 \\
7.4 \\
8.5
\end{tabular} & $\begin{array}{l}2.53 \\
2.63 \\
2.36 \\
2.19 \\
1.97 \\
1.59 \\
1.95\end{array}$ \\
\hline
\end{tabular}

m Mannitol clearance. Since the mannitol clearance is identical to the inulin clearance, inulin only is used in the headings.

A Average TmD figure used.

* Observations made by Goldring, Chasis, Ranges, and Smith.

$\dagger$ H. M. received $475 \mathrm{mgm}$. of progesterone from March 5 to March 12, 1941. 
TABLE III

Chronic diffuse glomerulonephritis

\begin{tabular}{|c|c|c|c|c|c|c|c|c|c|c|c|c|c|c|c|c|c|c|}
\hline Sub- & $\begin{array}{l}\text { Ante- } \\
\text { par- } \\
\text { tum } \\
\text { or } \\
\text { post- } \\
\text { par- } \\
\text { tum }\end{array}$ & $\begin{array}{l}\text { Sur- } \\
\text { face } \\
\text { area }\end{array}$ & Date & $\begin{array}{c}\text { Dura- } \\
\text { tion } \\
\text { of } \\
\text { preg- } \\
\text { nancy } \\
\text { by } \\
\text { his- } \\
\text { tory }\end{array}$ & $\begin{array}{l}\text { Time } \\
\text { be- } \\
\text { fore } \\
\text { or } \\
\text { after } \\
\text { de- } \\
\text { livery }\end{array}$ & $\begin{array}{l}\text { Blood } \\
\text { pres- } \\
\text { sure } \\
\text { during } \\
\text { test }\end{array}$ & $\begin{array}{c}\text { Protein- } \\
\text { uria }\end{array}$ & \multicolumn{3}{|c|}{$\begin{array}{c}\text { Plasma } \\
\text { clearances }\end{array}$} & $\begin{array}{l}\text { Effec- } \\
\text { tive } \\
\text { renal } \\
\text { blood } \\
\text { flow }\end{array}$ & $\begin{array}{c}\text { Inulin/ } \\
\text { Phenol } \\
\text { red }\end{array}$ & 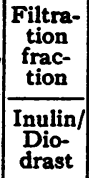 & $\begin{array}{l}\text { Phenol } \\
\text { red/ } \\
\text { Dio- } \\
\text { drast }\end{array}$ & $\underset{\text { Trast }}{\text { Dio- }}$ & $\begin{array}{c}\text { Effec- } \\
\text { tive } \\
\text { renal } \\
\text { blood } \\
\text { flow/ } \\
\text { T m }\end{array}$ & $\frac{C_{D} /}{T m_{D}}$ & $\begin{array}{l}\mathbf{C}_{\mathrm{ng}} / \\
\mathbf{T} \mathbf{m}_{\mathrm{D}}\end{array}$ \\
\hline P. R. & $\begin{array}{l}\text { A-P } \\
\mathbf{P}-\mathbf{P}\end{array}$ & $\begin{array}{l}\text { sq. } \\
\text { m. }\end{array}$ & $\begin{array}{l}\text { April 17, } 1941 \\
\text { September } 23,1941 \\
\text { January } 22,1942 \\
\text { July } 12,1943\end{array}$ & weeks & weeks & $\begin{array}{l}130 / 86 \\
168 / 110 \\
140 / 90 \\
130 / 80\end{array}$ & & $\begin{array}{l}79 \\
67 \mathrm{~m} \\
59 \mathrm{~m}\end{array}$ & $\begin{array}{l}237 \\
181\end{array} \mid$ & $\begin{array}{l}\text { m. } \\
\text { ute } \\
392 \\
424 \\
495 \\
319\end{array}$ & $\begin{array}{c}\text { ml. per } \\
1.73 \\
\text { sq. m. } \\
\text { per } \\
\text { minule } \\
628 \\
686 \\
800 \\
502\end{array}$ & $\begin{array}{l}\text { per } \\
\text { cent } \\
33.4 \\
32.6\end{array}$ & $\begin{array}{c}\text { per } \\
\text { cent } \\
\\
20.2 \\
15.8 \\
18.5\end{array}$ & $\begin{array}{l}\text { per } \\
\text { cent } \\
60.5 \\
56.7\end{array}$ & $\begin{array}{c}\text { mgm. } \\
\text { iodine } \\
\text { per } \\
\text { minute } \\
30.7 \\
40.0 \\
28.0\end{array}$ & $\begin{array}{l}20.5 \\
17.1 \\
17.9\end{array}$ & $\begin{array}{l}12.8 \\
10.6 \\
11.4\end{array}$ & $\begin{array}{l}2.57 \\
1.67 \\
2.10\end{array}$ \\
\hline J. Go. & $\begin{array}{l}\text { A-P } \\
\mathbf{P} \cdot \mathbf{P}\end{array}$ & 1.55 & $\begin{array}{l}\text { April } 9,1942 \\
\text { May 15, } 1942 \\
\text { July } 2,1942 \\
\text { September } 9,1942 \\
\text { July } 19,1943\end{array}$ & $\begin{array}{l}25 \\
29 \\
36\end{array}$ & $\begin{array}{r}15 \\
11 \\
4 \\
5\end{array}$ & $\begin{array}{l}156 / 114 \\
144 / 100 \\
200 / 135 \\
200 / 120 \\
180 / 120\end{array}$ & & $\begin{array}{l}62 \mathrm{~m} \\
60 \mathrm{~m} \\
52 \mathrm{~m} \\
53 \mathrm{~m} \\
\mathbf{4 8 m}\end{array}$ & $\begin{array}{l}198 \\
252 \\
181 \\
156 \\
144\end{array}$ & $\begin{array}{l}306 \\
420 \\
285 \\
260 \\
223\end{array}$ & $\begin{array}{l}504 \\
708 \\
473 \\
417 \\
415\end{array}$ & $\begin{array}{l}31.3 \\
23.8 \\
28.7 \\
34.0 \\
33.3\end{array}$ & $\begin{array}{l}20.3 \\
14.3 \\
18.2 \\
20.4 \\
21.5\end{array}$ & $\begin{array}{l}64.7 \\
59.9 \\
47.0 \\
59.5 \\
64.7\end{array}$ & $\begin{array}{l}39.8 \\
39.1 \\
30.2 \\
29.8 \\
26.7\end{array}$ & $\begin{array}{l}12.7 \\
18.1 \\
15.7 \\
14.0 \\
15.5\end{array}$ & $\begin{array}{r}7.7 \\
10.7 \\
9.4 \\
8.7 \\
8.4\end{array}$ & $\begin{array}{l}1.56 \\
1.53 \\
1.72 \\
1.78 \\
1.80\end{array}$ \\
\hline
\end{tabular}

m Mannitol clearance.

the drop in blood pressure which often occurs in normal pregnancy (15 to 17).

The permanent effect of pregnancy on the course of essential hypertension is a separate question, which is greatly confused by difficulties in classification of the vascular diseases of pregnancy. Considerable difference of opinion exists, but the prevailing view appears to be that pregnancy is detrimental to women with essential hypertension. However, it has been found (18) that pregnancy had no ill effects in 52 of 65 patients with pre-existing essential hypertension. Other authors (12) noted that of 89 patients followed for from 6 months to 3 years after delivery, 44 per cent had a blood pressure that was higher than when the patient was first seen, while among 28 patients followed for from 3 to 20 years, 20 had a blood pressure higher than on the first observation. These authors point out further that this incidence of blood pressure increase might be expected during such prolonged periods of observation, irrespective of pregnancy.

In the 7 pregnancies occurring in the 6 patients with essential hypertension in our series, there was no consistent rise in blood pressure, the variations from week to week being no greater than those noted before conception or after delivery, nor were there any other signs of superimposed toxemia. The 7 pregnancies resulted in the birth of healthy normal infants at term with 2 exceptions, each of the latter terminating in the birth of a stillborn premature infant. After labor, the elevation in blood pressure persisted, but there has been no evidence, after periods of post-partum observation of from 1 to 4 years, that the pregnancy has accelerated or otherwise altered the course of the disease.

\section{Renal function in relation to pregnancy with essen- tial hypertension}

The clearances of inulin or mannitol were within normal limits in all observations in the group with essential hypertension, whether these were made before, during, or after pregnancy. These patients were evidently at a stage of the disease when glomerular filtration had not yet been affected. - Neither the existence of pregnancy nor the after effects of pregnancy produced any alteration of this function.

Diodrast Tm, as studied in $\mathbf{5}$ of these patients, was also found to be normal throughout and thus likewise unaffected by the primary disease or by pregnancy or parturition.

The effective renal blood flow, as measured by the clearance of diodrast in 5 pregnancies and indicated by the phenol red clearance in the other 2, was found to be subject to what appear to be significant variations. Details of these tests may be seen in Table II. The changes in the $C_{D} / T_{D}$ ratio, giving the plasma flow per unit of excretory tubular tissue, can be seen more readily in Figure 1.

Before conception, the figure for the renal blood flow tended to be low in the 3 patients upon 


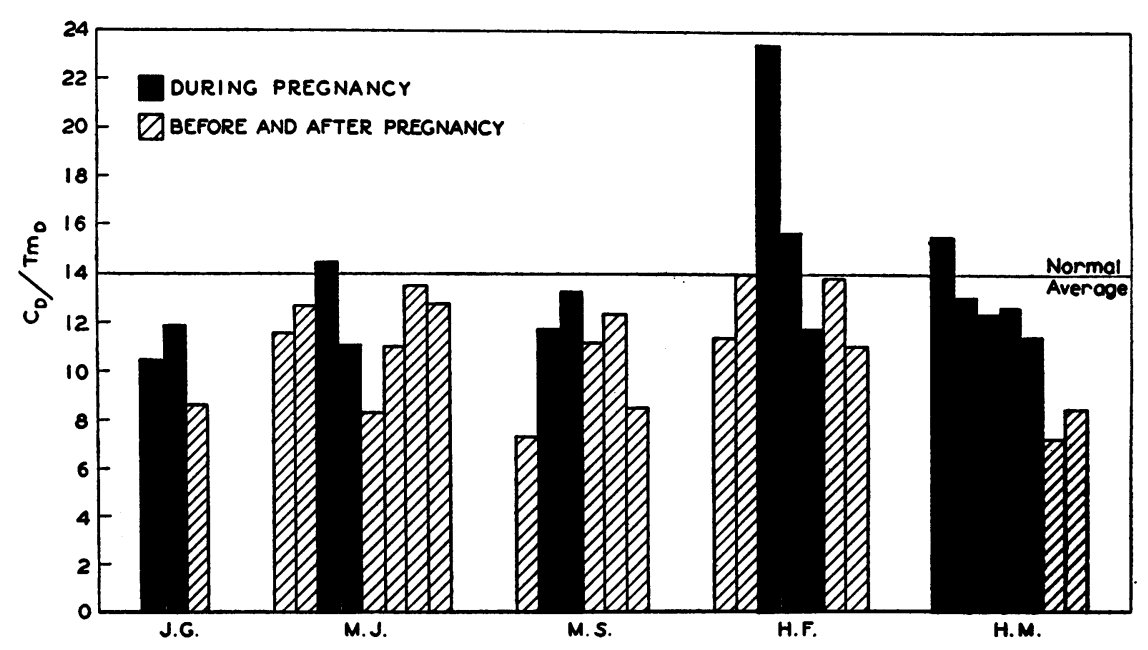

Fig. 1. Changes in Renal Plasma flow per Unit Ty Before, During, and After Pregnancy in Women with Essential Hypertension

whom observations were made at this time. Figures indicating a reduced renal blood flow are of course characteristic of patients with essential hypertension (8). In each of these, the diodrast clearance rose during their subsequent pregnancy.

After all of the 5 pregnancies studied by diodrast clearances, the blood flow was found also to be somewhat below normal. In each of these cases, there had been a higher average diodrast clearance and higher figures for the $\mathrm{C}_{\mathrm{D}} / \mathrm{Tm}_{\mathrm{D}}$ ratio (Figure 1 ) during their pregnancies than after their delivery. In 2 additional pregnancies studied only by the phenol red clearance, there was also found to be a higher figure for this function during pregnancy than after delivery. It may be significant that in 3 of the 7 pregnancies studied, the renal blood flow had fallen somewhat in the last observation made before delivery so that had these studies been limited to the last 3 weeks of pregnancy, the now apparent increase in blood flow during pregnancy might have been overlooked.

The filtration fraction $\left(C_{I N} / C_{D}\right)$ and the inulin/ phenol red clearance ratio are affected by these changes in renal blood flow. In general, these ratios were found high before conception and after delivery but approached the normal figure during pregnancy. On the basis of the significance placed upon the filtration fraction by some authors (20), the decrease in the filtration fraction which develops when hypertensive women become pregnant may be attributed to a diminished tone of the efferent arteriole from the glomerulus.

In the 3 patients in whom diodrast clearances were done both before and after pregnancy, there was no evidence of any diminution in renal blood flow as a result of the pregnancy. In 4 cases in which a series of clearance tests were made during a follow-up period, ranging from 1 to 4 years after delivery, there was no evidence of any progressive deterioration of renal function after pregnancy.

\section{Clinical effects of pregnancy on chronic diffuse glomerulonephritis}

The effect of pregnancy on chronic diffuse glomerulonephritis is considered by most obstetricians to be worse than on essential hypertension. One author (14) writes that it is unusual for a patient with chronic diffuse gomerulonephritis to give birth to a live baby at term, or even for the pregnancy to continue to term. Others (18) believe that about half of their 17 patients with this disease became worse as a result of their pregnancies. A somewhat more optimistic report has been given (12) on the basis of 15 cases. Among these, the blood pressure increased during pregnancy in 8 and the proteinuria increased in 7 . From 6 months to 5 years after delivery, 4 patients were worse, 2 being in a serious condition. The usual course of the disease, uncomplicated by pregnancy, 
might, however, give results not dissimilar from these.

The 2 patients with chronic diffuse glomerulonephritis in our group completed their pregnancies and were delivered at term of normal children without increase of either their hypertension or proteinuria. Twenty-eight months have elapsed since the delivery of 1 of these patients and 16 since that of the other. Both continue to show evidence of active glomerulonephritis with hypertension, proteinuria, and hematuria, but in neither is there evidence of significant progression of the renal lesion as the result of the pregnancies.

\section{Renal function in relation to pregnancy and chronic glomerulonephritis}

The 2 patients with chronic glomerulonephritis were known to have had diminished urea clearances before conception. At the time they were first examined by the more complete clearance methods, they were already pregnant. In each, there were then noted abnormally low values for the glomerular filtration, renal blood flow, and tubular excretory mass. That the glomerular filtration rate and renal blood flow were impaired to a greater extent than the tubular excretory mass can be seen from the low values of $C_{I n} / T_{m}$ and of $C_{D} / T_{m}$.

On the basis of only 2 cases, no consistent difference can be pointed out between antepartum and post-partum values, so that in this disease pregnancy appears to have had no effect on renal blood flow. This, in distinction to the alterations noted in cases of essential hypertension, might be due to the more permanent character of the changes causing disturbances in function in the kidney with chronic nephritis.

When comparing the values obtained in the observations made during pregnancy witn those made 1 and 2 years, respectively, after the delivery of the 2 women, one finds a suggestion of slight diminution in all functions measured. These changes are not striking, perhaps not significant, but are consistent with the slowly progressive course which is usually characteristic of the disease. There is no evidence, however, of an abrupt change in the course resulting from pregnancy.

\section{DISCUSSION}

Two points are of special interest. First, it is necessary to consider the significance of the apparent increase in renal blood flow which developes when the woman with essential hypertension becomes pregnant. Secondly, consideration must be given the clinical implications of the renal function tests made before, during, and at intervals after a pregnancy complicated by pre-existing renal or vascular disease.

The increase in the renal blood flow during pregnancy, as indicated by the diodrast and phenol red clearance, appears to be real, although slight in degree. In each of the 3 patients upon whom diodrast clearance observations were made before conception, the figures for blood flow were found to have risen during pregnancy. Similarly, in all 7 patients, it could be shown that the blood flow fell after delivery.

This behavior of the renal blood flow in cases of essential hypertension is parallelled by observations made on patients with specific toxemia (2). In the latter condition, the renal blood flow is normal or slightly elevated during pregnancy, but in those patients who develop a persistent hypertension, evidences of relative renal ischemia appear immediately after parturition.

These results may have some bearing on the effects of pregnancy upon blood pressure. In experimental animals, previously rendered hypertensive by constriction of the aorta or renal artery, there has been observed a decrease in the level of hypertension with the advent of pregnancy (21 to 25). Furthermore, if the operation for the reduction of the renal blood flow is carried out after pregnancy has commenced, the rise in blood pressure is frequently delayed till after delivery (25).

No evidence can be found in a review. of the records of our patients of even a transitory decline in blood pressure. Other observers (12) have noted, however, that in a considerable proportion of patients with essential hypertension there occurs a rather "striking" decrease in the blood pressure during the middle trimester of pregnancy. The opposite effect, namely a temporary rise in blood pressure for several weeks after the conclusion of a normal pregnancy, has recently been described with reports of a considerable number of such cases $(27,28)$. 
Speculation on the cause of the improved renal blood flow noted in pregnant hypertensive patients leads at once to a consideration of the placenta. The sudden contraction of the total vascular bed through the elimination of the large circulation of the maternal portions of the placenta might conceivably affect blood pressure for a short time but it is difficult to see how renal circulation could be affected by this means. Evidence that any hormones of placental origin have a direct effect on renal blood vessels has not been produced, but it is known that the estrogens and perhaps other sex steroid hormones have some effects on the peripheral circulation (29). It is at least possible that the blood flow of the kidney in hypertensive pregnant patients is maintained by some such substance from the placenta whose loss at delivery is at once followed by relative renal ischemia.

The failure to find signs of any deterioration in renal function after pregnancy in women with essential hypertension or chronic glomerulonephritis is a point of practical importance in the handling of these patients. These observations run somewhat contrary to existing beliefs.

Satisfactory statistics showing the effect of pregnancy on pre-existing essential hypertension and chronic glomerulonephritis are meagre and confusing due to lack of agreement on the criteria for classifying cases. It is agreed that an attack of specific toxemia of pregnancy may have chronic vascular disease as a sequela and that in cases of essential hypertension, a "superimposed" toxemia is liable to occur. It may be inferred that an attack of toxemia in a patient already suffering from essential hypertension or chronic glomerulonephritis will be detrimental. However, it is also evident that patients with these diseases may go through their pregnancies without added complications and our studies indicate that pregnancy without superimposed toxemia has no permanent effects on the renal function of women suffering from chronic vascular or renal diseases.

\section{CONCLUSIONS}

1. Renal function studies made upon patients with essential hypertension indicate that pregnancy is associated with a slight temporary increase in renal blood flow. Glomerular filtration rate and the tubular excretory masis (Diodrast $\mathrm{Tm})$ are unaffected by pregnancy in these women.

2. Comparison of results obtained duringpregnancy and for an observation period of 1 to 4 years after delivery indicate that pregnancy itself, when uncomplicated by specific toxemia, does not cause any deterioration of renal function in women with essential hypertension or chronic glomerulonephritis.

\section{BIBLIOGRAPHY}

1. Welsh, C. A., Wellen, I., and Taylor, H. C., Jr., The filtration rate, effective renal blood flow, tubular excretory mass and phenol red clearance in normal pregnancy. J. Clin. Invest., 1942, 21, 57.

2. Wellen, I., Welsh, C. A., and Taylor, H. C., Jr., The filtration rate, effective renal blood flow, tubular excretory mass and phenol red clearance in specific toxemia of pregnancy. J. Clin. Invest., 1942, 21, 63.

3. Corcoran, A. C., and Page, I. H., Renal function in the late toxemia of pregnancy. Am. J. M. Sc., 1941, $201,385$.

4. Dill, L. V., Isenhour, C. E., Cadden, J. F., and Schaffer, N. K., Glomerular filtration and renal blood flow in the toxemias of pregnancy. Am. J. Obst. and Gynec., 1942, 43, 32.

5. Smith, W. W., Finkelstein, N., and Smith, H. W., Renal excretion of hexitols (sorbitol, mannitol and dulcitol) and their derivatives (sorbitan, isomannide and sorbide) and of endogenous creatinine-like chromogen in dog and man. J. Biol. Chem., 1940, 135, 231.

6. Smith, H. W., Goldring, W., and Chasis, H., The measurement of the tubular excretory mass, effective blood flow, and filtration rate, in the normal human kidney. J. Clin. Invest., 1938, 17, 263.

7. Goldring, W., Chasis, H., Ranges, H. A., and Smith, H. W., Relations of effective renal blood flow and glomerular filtration to tubular excretory mass in normal man. J. Clin. Invest., 1940, 19, 739.

8. Smith, H. W., Lectures on the Kidney. William Henry Welch Lectures, Waverly Press, Baltimore, 1943.

9. Dexter, L., and Weiss, S., Pre-eclamptic and Eclamptic Toxemia of Pregnancy. Little, Brown and Company, Boston, 1941.

10. Herrick, W. W., and Tillman, A. J. B., Toxemia of pregnancy; its relation to cardiovascular and renal disease; clinical and necropsy observations with a long follow-up. Arch. Int. Med., 1935, 55, 643.

11. Heynemann, T., Spätfolgen der Eklampsie und ihrer Vorstadien unter besonderer Berücksichtigung der Nierenveränderungen. Zentralbl., f. Gynäk., 1934, 58, 3010.

12. Reid, D. E., and Teel, H. M., Non-convulsive pregnancy toxemias. Their relationship to chronic 
vascular and renal disease. Am. J.'Obst. and Gynec., 1939, 37, 886.

13. Wellen, I., Specific "toxemia," essential hypertension, and glomerulonephritis associated with pregnancy. Am. J. Obst. and Gynec., 1940, 39, 16.

14. Dieckmann, W. J., The Toxemias of Pregnancy. The C. V. Mosby Company, St. Louis, 1941.

15. Hamilton, B. E., and Thomson, K. J., The Heart in Pregnancy and the Childbearing Age. Little, Brown and Company, Boston, 1941.

16. Hare, D. C., and Karn, M. N., An investigation of blood pressure, pulse rate and response to exercise during normal pregnancy and some observations after confinement. Quart. J. Med., 1929, 22, 381.

17. Danforth, W. C., Results of blood pressure. Observations in 447 cases of pregnancy. Am. J. Obst., 1918, 77, 927.

18. Browne, F. J., and Dodds, G. H., The remote prognosis of toxaemias of pregnancy based on a follow-up study of $\mathbf{4 0 0}$ patients in $\mathbf{5 8 9}$ pregnancies for periods varying from 6 months to 12 years. J. Obst. and Gynaec. Brit. Emp., 1939, 46, 443.

19. Goldring, W., Chasis, H., Ranges, H. A., and Smith, H. W., Effective renal blood flow in subjects with essential hypertension. J. Clin. Invest., 1941, 20, 637.

20. Smith, H. W., Chasis, H., Goldring, W., and Ranges, H. A., Glomerular dynamics in the normal human kidney. J. Clin. Invest., 1940, 19, 751.

21. Goldblatt, H., Kahn, J. R., and Hanzal, R. F., Studies on experimental hypertension. IX. The effect on blood pressure of constriction of the abdominal aorta above and below the site of origin of both main renal arteries. J. Exper. Med., 1939, 69, 649.

22. Harrison, T. R., Grollman, A., and Williams, J. R., Jr., The antipressor action of renal extracts and their capacity to reduce the blood pressure of hypertensive rats. Am. J. Physiol., 1940, 128, 716.

23. Foa, P. P., Foa, N. L., and Peet, M. M., Effect of pregnancy on experimental renal hypertension in rats. Am. J. M. Sc., 1942, 204, 350.

24. Corbit, J. D., Jr., The effect of pregnancy upon experimental hypertension in the rabbit. Am. J. M. Sc., 1941, $201,876$.

25. Page, E. W., Patton, H. S., and Ogden, E., The effect of pregnancy on experimental hypertension, with observations on the effects of deciduomas. Am. J. Obst. and Gynec., 1941, 41, 53.

26. Dill, L. V., Isenhour, C. E., and Cadden, J. F., The effect of quantitative reduction of renal blood flow upon the pregnant rabbit. J. Clin. Invest., 1939, 18, 641.

27. Meyer, H., and Nadler, S. B., Unexpected postpartum hypertension. Am. J. Obst. and Gynec., 1941, 41, 231.

28. Stout, M. L., Hypertension six weeks postpartum in apparently normal patients. Am. J. Obst. and Gynec., 1934, 27, 730.

29. Reynolds, S. R. M., Physiology of the Uterus with Clinical Correlations. Paul B. Hoeber, Inc., New York, 1939. 